\title{
Es braucht neue
}

\section{Vergütungsmodelle}

\author{
Johannes Spalinger \\ Dr. med., Pädiatrische Klinik Kinderspital Luzern
}

1 Genewein A, Hänsenberger S. Nichtärztlicher Personalaufwand. Schweiz Ärztezeitung 2016;97(45):1573-6.

Korrespondenz: Dr. med. Johannes Spalinger Mitglied Fachteam Kind TARMED

Schweiz. Gesellschaft für Pädiatrie

Co-Chefarzt Pädiatrische Gastroenterologie Kinderspital Luzern CH-6000 Luzern johannes.spalinger[at] luks.ch
Kindermedizin bedeutet kindergerechte, einfühlsame und möglichst wenig traumatisierende medizinische Betreuung vom Säugling bis zum Adoleszenten. Es betrifft eine Patientengruppe, welche sehr verletzlich ist, bei der besondere emotionale Bedingungen herrschen und vielfach eine Kooperation selbst bei kleinen Eingriffen nicht erwartet werden kann.

Eine kindermedizinische Versorgung soll kindergerecht, nicht traumatisierend, unter Einbezug der Eltern und natürlich fachlich-technisch auf höchstem Niveau erfolgen. Ein wesentlicher Beitrag zur Versorgung von Kindern und ihren Familien leistet das nichtärztliche Personal. Die detaillierte Analyse von Genewein et al. dokumentiert einen erheblichen Aufwand durch das Pflegepersonal, welcher im aktuellen Tarifsystem nicht abgebildet wird.

Die Notfallstationen und Ambulatorien der Schweizer Kinderspitäler und Kinderabteilungen stehen aktuell unter enorm hohem Druck, denn die bestehenden Tarifstrukturen verunmöglichen es, kostendeckende Leistungen zu erbringen. Der 24-Stunden-Betrieb der Notfallstationen mit den grossen Vorhalteleistungen und dem hohen Personalaufwand ist defizitär. Dieser unbefriedigende Zustand wird durch den anhaltenden Trend der starken Zunahme an Notfallkonsultationen (insbesondere abends, nachts, an Wochenenden und Feiertagen) und der Nachfrage an ambulanten Leistungen (Kleineingriffe, Narkoseuntersuchungen) noch verschärft.

In den meisten ambulanten Stationen (Notfallstationen, Tageskliniken) ist das Pflegepersonal für eine korrekte Vorerfassung (Triage) und Beurteilung der Situation (Gesundheitsstatus) zuständig. Die Vorbereitung auf einen Kleineingriff (z.B. Prämedikation) und die Begleitung vor, während und nach einem Eingriff haben bei der Versorgung von Kindern einen viel höheren Stellenwert als bei Erwachsenen.

Die Annahme, dass nur Kleinkinder einen höheren Betreuungsaufwand durch die Pflege benötigen, wird in der Studie widerlegt: Selbst 14-Jährige sind aus Angst weniger kooperativ und benötigen eine vermehrte Zuwendung durch die Pflege. Die Autoren kommen zum Schluss, dass Kinder generell von 0- bis 16-jährig als zuschlagsberechtigt gelten.
Da die spezialisierte ambulante Kindermedizin mit Kleineingriffen, invasiver Diagnostik (Untersuchungen in Sedation oder Narkose) praktisch ausschliesslich in Kinderspitälern stattfindet und diese nicht kostendeckend durchgeführt werden kann, sind zwingend neue Vergütungsmodelle zu erarbeiten. Nur so kann langfristig den Bedürfnissen einer kindergerechten und fachlich hochstehenden Versorgung der Kinder entsprochen werden.

Der Vorschlag der Autoren, durch Notfall-Pauschalen und Nachtzuschläge eine kostendeckende Kinder-Notfallmedizin zu betreiben ohne dabei Anreize zur Verlagerung aus der Arztpraxis ins Spital zu schaffen, scheint ein guter Ansatz zur Verbesserung der Notfallversorgung. Allerdings ist die bestehende Tarifstruktur auch nicht kostendeckend für nichtnotfallmässige Kleineingriffe in Kinder-Ambulatorien oder Tageskliniken, so dass auch in diesem Bereich Anpassungen dringend erforderlich sind.

Die im ersten Anlauf gescheiterte TARMED-Revision zeigte gute Ansätze, nicht nur den grösseren Zeitaufwand der ärztlichen Versorgung, sondern auch den personellen Mehrbedarf bei der Pflege bzw. dem nichtärztlichen Personal korrekter abzubilden.

Das Unbehagen der Praxisärzte am ungebremsten Ausbau der Spitalambulatorien ist verständlich, allerdings steht die Kindermedizin diesbezüglich weniger im Fokus, da es sich in der Regel um "Zuweisungsambulatorien» handelt und ein freier Zugang nicht möglich ist. Zudem sind die Notfallstationen der Kinderspitäler zunehmend die erste Anlaufstelle bei Notfällen und Unfällen, insbesondere abends, nachts, an Wochenenden und Feiertagen.

Die in der Studie erhobenen Zahlen, die erhebliche Unterdeckung der ambulanten kindermedizinischen Leistungen im nichtärztlichen Personalaufwand, aber auch bei den ärztlichen Leistungen, sind wichtige Grundlagen, welche in die neu aufgegleiste Tarifdiskussion (TARCO) mit den Fach- und Dachgesellschaften einfliessen müssen. Nur so kann eine kostendeckende medizinische Versorgung der Kinder auch in Zukunft gewährleistet werden. 